\title{
Incidencia de los factores económicos-sociales en el impulso de la gestión de la competitividad nacional del Perú - periodo 2000-2014
}

Incidence of economic-social factors in the impulse of the management of national competitiveness of Peru - period 2000-2014

Nelson Cruz Castillo ${ }^{1}$

\section{RESUMEN}

Determinar de qué manera inciden los factores económicos-sociales en el impulso de la gestión de la competitividad nacional del Perú, que está representado por la productividad como variable dependiente. Estos factores son siete, por lo que, se plantea siete objetivos específicos, los cuales algunos de ellos están limitados por la escasez de datos que fueron extraídos principalmente del banco Mundial y del INEI. Por un lado, la contrastación de la hipótesis general, realizado por el análisis de regresión múltiple, nos dio como resultado, que los factores desempeño económico, educación y salud tienen incidencia positiva en la variable dependiente; por otro lado, los factores infraestructura, institucionalidad, innovación, educación a través de la variable inscripción escolar en el nivel secundario y clima de negocios tienen incidencia negativa sobre la variable dependiente. Se aplica el método de los MCO. En el análisis econométrico con logaritmos, se refleja que las variables independientes inciden de diferentes maneras sobre el comportamiento de la variable dependiente. Así, considerando la contrastación de las hipótesis específicas, se concluye que, la importancia de la incidencia del factor infraestructura no es muy significativa; la influencia del factor desempeño económico si es relevante; la incidencia del factor institucional no es muy significativa; el factor innovación no tiene una importante incidencia; la influencia del factor educación si es significativamente importante; el factor salud si contribuye de manera muy importante; y el impacto del factor clima de negocios es negativo pero no significativa en la variable dependiente. En consecuencia los coeficientes son estadísticamente significativos.

Palabras clave: competitividad nacional; productividad; factores económico-sociales; gestión.

1 Universidad Nacional Mayor de San Marcos. Lima, Perú. 


\section{ABSTRACT}

Determine how economic-social factors affect Peru's national competitiveness management, which is represented by productivity as a dependent variable. These factors are seven, so seven specific objectives were proposed, some of them limited by the scarcity of data that were extracted mainly from the World Bank and INEI. On the one hand, the comparison of the general hypothesis, carried out by the multiple regression analysis, gave us as a result, that the economic performance factors, education and health have a positive effect on the dependent variable. On the other hand, factors infrastructure, institutionality, innovation, education through the variable school enrollment in the secondary level and business climate have a negative impact on the dependent variable. Is applied, according to the $\mathrm{MCO}$ method. In the econometric analysis with logarithms, it is reflected that the independent variables affect in different ways on the behavior of the dependent variable. Thus, considering the contrasting of the specific hypotheses, it was concluded that, the importance of the incidence of the infrastructure factor is not very significant; the influence of the economic performance factor if relevant; the incidence of the institutional factor is not very significant; the innovation factor does not have a significant impact; the influence of the education factor if it is significantly important; the health factor contributes in a very important way; and the impact of the business climate factor is negative but not significant in the dependent variable. Consequently the coefficients are statistically significant.

Keywords: national competitiveness; productivity; economic-social factors; management.

\section{INTRODUCCIÓN}

A nivel global ya se han hecho diversos estudios sobre el tema de la competitividad de las naciones o de los países, tal es el caso del Índice de Competitividad Global elaborado por el Foro Económico Mundial (2012), y a nivel nacional realizado por el Instituto Peruano de Economía (2013) y el Consejo Nacional de Competitividad.

Pero estas investigaciones o reportes antes mencionados no han tomado en cuenta, el método y la técnica generalmente utilizados por los economistas, como es la econometría. Es decir, la metodología de investigación que se aplicó es diferente a los que generalmente nos han presentado algunas instituciones. En el presente estudio se pretendió confirmar si los factores institucionalidad, innovación, infraestructura, desempeño económico, salud, educación y el clima de negocios inciden en el impulso de la gestión de la competitividad nacional del Perú. Entonces es relevante describir las causas por las cuales el 
Perú no alcanza mejores niveles de competitividad. En consecuencia, se debe tener en cuenta las posibles soluciones para aquellos problemas que le restan competitividad. La principal fuente de información de los factores económicos-sociales es el Instituto Nacional de Estadística e Informática (INEI) del Perú, así como del Consejo Nacional de Competitividad y del Banco Mundial. Así, entonces convirtiendo esta información en conocimiento se ha tenido un consistente indicador de competitividad, que es la productividad, que servirá para el planteamiento y desarrollo de las estrategias y políticas nacionales.

El estudio permite por un lado, hallar recomendaciones para mejorar la gestión de los recursos y capacidades de la nación peruana; y por otro lado, aplicar una metodología diferente a lo aplicado por otras instituciones o autores. En ese sentido presentamos en primer lugar la metodología aplicada y las variables intervinientes, después se realiza el análisis económico y la aplicación de las técnicas econométricas, y finalmente se realiza las contrastaciones empíricas y teóricas de los resultados los que nos lleva a plantear las respectivas conclusiones.

\section{MATERIALES Y MÉTODOS}

Según su finalidad: es aplicada y es no experimental, ya que se trata de aportar y resolver problemas de naturaleza práctica. Según su naturaleza: es explicativa porque analiza y explica los factores económico - sociales que inciden en la gestión de la competitividad nacional del Perú. Se utilizó información estadística de un grupo de indicadores económicos y no económicos del Perú. Según el tiempo de estudio es de tipo longitudinal, ya que se recolectaron datos en el periodo de tiempo $2000-2014$.

El diseño de la presente investigación se realizó sin manipular deliberadamente las variables o conceptos. El tipo de investigación es explicativo - correlacional, utilizando las técnicas econométricas por que los estudios nos presentan un panorama del estado de las variables en uno o más grupos de indicadores en determinado momento. El nivel de investigación es exploratorio, dado que según el estado de conocimiento de la investigación sobre los factores económicos sociales que impulsan la competitividad nacional del Perú es escaso o casi nulo, según el uso de las técnicas econométricas. Población de estudio: para la determinación de la población de la que se extrajo la muestra, se considera a los sectores productivos del país y a las entidades del sector público. Para la determinación de la muestra, se consideró a los sectores productivos del país y a las entidades del sector público.

El procesamiento del análisis de los datos es de orden cualitativo, debido a que se tuvo valores de tipo económico y no económico desde lo simple a lo 
complejo. El proceso de elaboración de un modelo econométrico parte del planteamiento, o existencia inicial de una hipótesis o ley de comportamiento (teoría) que debemos contrastar frente a un conjunto de datos o realizaciones observadas concretas.

Así, se ha planteado un modelo econométrico de acuerdo con la siguiente expresión: $\mathrm{Yt}=\mathrm{a}+\mathrm{bXt}+\mathrm{Zt}$

A partir de los $n$ datos del periodo muestral elegido $(t=1,2,3, \ldots, N)$, el conocimiento de los coeficientes a, b, c, d, e, f, g, h nos permite realizar las siguientes aplicaciones y análisis: análisis estructural, predicción, evaluación de políticas, proceso científico de toma de decisiones. En ese sentido el presente estudio aplicó el Modelo Básico de Econometría, que permite explicar el comportamiento de una (o varias) variable endógena mediante una relación lineal de $\mathrm{K}$ variables explicativas, con un término de error aditivo o perturbación aleatoria que se supone cumplen las características de un ruido blanco.

Especificación de un modelo lineal simple:

$$
\mathrm{Y}=\mathrm{a}+\mathrm{bX} 1+\mathrm{cX} 2+\mathrm{dX} 3+\mathrm{eX} 4+\mathrm{fX} 5+\mathrm{gX} 6+\mathrm{hX} 7+\mathrm{u}
$$

Donde; $\mathrm{Y}=\mathrm{La}$ gestión de la competitividad nacional del Perú.

Los factores económicos sociales son:

X1 = Infraestructura; X2 = Desempeño Económico; X3 = Institucionalidad;

X4 = Innovación; X5 = Educación; X6 = Salud; X7 = Clima de Negocios

Tenemos la expresión: $\mathrm{Y}=\mathrm{XB}+\mathrm{u}$

En cuanto a la presente investigación, el análisis del modelo se hizo en forma general como se indica en la hipótesis general; asimismo, se realizó la contrastación de las hipótesis específicas. Formulación del Modelo Econométrico:

\section{Hipótesis General}

A partir del modelo anterior se especificó el siguiente modelo econométrico para su correspondiente estimación, que recoge los indicadores representativos de cada variable:

$\mathrm{Y}=\beta \mathrm{O}+\beta 1 \mathrm{X} 1 \mathrm{~A}+\beta 2 \mathrm{X} 1 \mathrm{~B}+\beta 3 \mathrm{X} 2 \mathrm{~A}+\beta 4 \mathrm{X} 2 \mathrm{~B}+\beta 5 \mathrm{X} 3 \mathrm{~A}+\beta 6 \mathrm{X} 3 \mathrm{~B}+\beta 7 \mathrm{X} 4$

$+\beta 8 \mathrm{X} 5 \mathrm{~A}+\beta 9 \mathrm{X} 5 \mathrm{~B}+\beta 10 \mathrm{X} 6+\beta 11 \mathrm{X} 7+\mu \mathrm{i}$

Donde: 
$\mathrm{Y}=$ Productividad nacional del Perú (PBI/ PEA ocupada)

$\mathrm{X} 1 \mathrm{~A}=$ Hogares que se abastecen de agua.

$\mathrm{X} 1 \mathrm{~B}=$ Hogares que acceden al servicio de internet.

$\mathrm{X} 2 \mathrm{~A}=$ PBI per cápita $(\mathrm{S} /$. de 2007)

$\mathrm{X} 2 \mathrm{~B}=$ Inflación (IPC).

X3A = Inversión per cápita del gobierno nacional (en nuevos soles de 2007).

$\mathrm{X} 3 \mathrm{~B}=$ Eficacia presupuestal en inversiones del gobierno nacional.

X4 = Artículos en Publicaciones científicas y técnicas.

$\mathrm{X} 5 \mathrm{~A}=$ Inscripción escolar en el nivel secundario

$\mathrm{X} 5 \mathrm{~B}=$ Población activa con educación superior.

X6 = Esperanza de vida al nacer (años de vida)

$\mathrm{X} 7$ = Costo de los procedimientos para establecer una empresa.

$\beta_{\mathrm{o}}=$ constante paramétrica a estimar.

$\beta \mathrm{i}=$ Coeficientes de las variables explicativas $(i=1,2,3,4,5,6)$

$\mu \mathrm{i}=$ termino de perturbación estocástica.

Este término de perturbación puede representar claramente a todos aquellos factores que afectan a la gestión de la competitividad nacional, pero que no son considerados en el modelo en forma explícita. Es necesario remarcar que el indicador de la productividad es el PBI entre la Población Económicamente Activa, y que uno de los indicadores relevantes del factor desempeño económico es el PBI entre la cantidad total de la población, es decir, el PBI per cápita.

\section{RESULTADOS}

$\mathrm{Al}$ analizar los factores que inciden en el impulso de la gestión de la competitividad nacional del Perú, examinamos la unidad de muestreo cuyos datos se expresan en la tabla 1 .

El indicador de la gestión de la competitividad nacional del Perú es la productividad. Podemos observar que la productividad está en nuevos soles constantes, y si en el año 2000 fue de 18,607 nuevos soles, entonces el crecimiento porcentual de la productividad entre el año 2000 y 2014 fue de 48,1\%. Del año 2002 hasta el 2006, el PBI crece sostenidamente, aunque la PEA ocupada había disminuido hasta el 2004 y empieza aumentar a partir del 2005. Desde el año 2007 al 2014, el crecimiento promedio anual del PBI fue de 6,2\%. A nivel 
global la crisis de los Estados Unidos ya se estaba extendiendo, por lo que, el PBI del Perú empieza una tendencia declinatoria llegando a crecer solamente 2,4\% en el 2014. En el periodo 2007-2014 la productividad crece un 25,7\%, mientras que el PBI lo hace en 49,9\%. Así, en estos años el consumo público crece $66,2 \%$, que viene a ser $19 \%$ más que el consumo privado. El mayor crecimiento de la inversión pública fue en el año 2008, con 33,7\% y el de la inversión privada también fue en ese año, con 25,9\%. Aunque ya apreciamos que la inversión interna cae sostenidamente a partir del año 2014, con una variación porcentual real de $-20,6 \%$.

A partir del 2006 la PEA empieza a recuperar su crecimiento. En el año 2000 esta llega alrededor de 12 millones de personas, y en el 2014 llega alrededor de los 17 millones de personas.

Tabla 1. Análisis de los factores económicos - sociales

\begin{tabular}{|c|c|c|c|c|c|c|c|c|c|c|c|c|}
\hline Años & $\begin{array}{c}\text { Produc- } \\
\text { tividad } \\
\text { Nacional } \\
\text { (PBI / } \\
\text { PEA) } \\
\text { (nuevos } \\
\text { soles a } \\
\text { precios } \\
\text { constantes } \\
\text { del 2007) } \\
\end{array}$ & $\begin{array}{l}\text { Hogares } \\
\text { que se } \\
\text { abaste- } \\
\text { cen de } \\
\text { agua (\% } \\
\text { respecto } \\
\text { del total) }\end{array}$ & $\begin{array}{l}\text { Hogares } \\
\text { que } \\
\text { acceden } \\
\text { al ser- } \\
\text { vicio de } \\
\text { internet } \\
\text { ( } \% \text { res- } \\
\text { pecto del } \\
\text { total) }\end{array}$ & $\begin{array}{l}\text { Producto } \\
\text { Bruto In- } \\
\text { terno por } \\
\text { habitante } \\
\text { (a precios } \\
\text { constan- } \\
\text { tes de } \\
2007 \text { ), } \\
\text { nuevos } \\
\text { soles }\end{array}$ & $\begin{array}{l}\text { Índice de } \\
\text { precios al } \\
\text { consumidor } \\
(2010= \\
100)\end{array}$ & $\begin{array}{c}\text { Inver- } \\
\text { sión per } \\
\text { cápita del } \\
\text { gobierno } \\
\text { nacional } \\
\text { (a precios } \\
\text { constan- } \\
\text { tes de } \\
2007 \text { ), } \\
\text { soles } \\
\end{array}$ & $\begin{array}{c}\text { Eficacia } \\
\text { presu- } \\
\text { puestal } \\
\text { en } \\
\text { inversio- } \\
\text { nes del } \\
\text { gobierno } \\
\text { nacional } \\
\text { (en } \%)\end{array}$ & $\begin{array}{l}\text { Artículos } \\
\text { en publi- } \\
\text { caciones } \\
\text { científicas } \\
\text { y técnicas }\end{array}$ & $\begin{array}{l}\text { Inscrip- } \\
\text { ción } \\
\text { escolar, } \\
\text { nivel se- } \\
\text { cundario } \\
\text { (\%bruto) }\end{array}$ & $\begin{array}{l}\text { Población } \\
\text { activa con } \\
\text { educación } \\
\text { superior ( } \% \\
\text { del total) }\end{array}$ & $\begin{array}{c}\text { Esperanza } \\
\text { de vida } \\
\text { al nacer } \\
\text { (años) }\end{array}$ & $\begin{array}{c}\text { Costo de } \\
\text { los proce- } \\
\text { dimientos } \\
\text { para esta- } \\
\text { blecer una } \\
\text { empresa } \\
(\% \text { del } \\
\text { INB per } \\
\text { cápita) }\end{array}$ \\
\hline 2000 & 18,607 & 63 & 0.1 & 8,552 & 79.161854 & 163.4 & 79.7 & 125.7 & 84.69558 & 29.8999996 & 70.51 & 43 \\
\hline 2001 & 17,579 & 63.6 & 0.5 & 8,480 & 80.726962 & 185.6 & 86.3 & 128.1 & 87.55472 & 29.6000004 & 70.95 & 41 \\
\hline 2002 & 18,720 & 64.9 & 0.9 & 8,817 & 80.882874 & 161.6 & 82 & 128.5 & 88.64472 & 30 & 71.37 & 41 \\
\hline 2003 & 19,687 & 62.2 & 1.5 & 9,061 & 82.7103235 & 147.8 & 35.2 & 187 & 81.40194 & 33 & 71.75 & 39.4 \\
\hline 2004 & 20,332 & 62.5 & 2.1 & 9,387 & 85.739567 & 120.9 & 123 & 194.4 & 84.5791 & 33.7999992 & 72.1 & 36.4 \\
\hline 2005 & 21,268 & 62.6 & 3.7 & 9,851 & 87.1253772 & 126.3 & 77.2 & 216.9 & 85.44732 & 11.8000002 & 72.42 & 38 \\
\hline 2006 & 21,955 & 64.3 & 5.1 & 10,465 & 88.8698521 & 114.1 & 67 & 284.3 & 88.07086 & 12 & 72.72 & 31.1 \\
\hline 2007 & 21,925 & 63.3 & 6.6 & 11,224 & 234 & 118 & 64.1 & 290.6 & 896 & 14.6000004 & 73.01 & 28.3 \\
\hline 2008 & 23,219 & 63.5 & 8.6 & 12,111 & 95.685148 & 121.9 & 53.1 & 345.4 & 91.04757 & 14 & 73.31 & 24.4 \\
\hline 2009 & 22,817 & 65.8 & 11.0 & 12,107 & 98.4946854 & 201.1 & 80.3 & 397.5 & 93.40124 & 15.1000004 & 73.6 & 16.2 \\
\hline 2010 & 24,229 & 67.9 & 13.0 & 12,969 & 100 & 256.1 & 81 & 466.8 & 94.70003 & 15 & 73.91 & 12.7 \\
\hline 2011 & 25,270 & 68.6 & 16.4 & 13,634 & 103.370685 & 260.7 & 81 & 545.7 & 95.20703 & 15.1000004 & 74.21 & 11.1 \\
\hline 2012 & 26,355 & 75 & 20.2 & 14,308 & 107.147573 & 227.3 & 82 & 563.9 & 93.43105 & 22.8999996 & 74.52 & 9.9 \\
\hline 2013 & 27,408 & 76.3 & 22.1 & 14,989 & 110.165388 & 267.2 & 81 & 647.8 & 97.89767 & 23.1000004 & 74.82 & 9.5 \\
\hline 2014E/ & 27,553 & 79.3 & 23.5 & 15,177 & 113.719375 & 313.8 & 88 & 717 & 95.62184 & 23.6000004 & 75.1 & 9.2 \\
\hline
\end{tabular}

Fuente: Banco Mundial y el INEI-Perú

En el año 2000 el 63\% de hogares en el Perú se abastecían de agua, lo cual describe la insuficiencia en infraestructura en este sector. Es a partir del año 2009 que empieza a aumentar la cantidad de hogares que tienen agua, llegando a 79,3\% de hogares en el año 2014. Entre los años 2000 y 2002, los hogares que accedían a la Internet no superaban el 1\% respecto del total de hogares. Para el año 2014, el 23,5\% de hogares a nivel nacional tienen acceso a Internet. Del año 2000 al año 2014, el PBI aumentó en 245,489 millones de nuevos soles, es 
decir, el aumento fue de $110.46 \%$. Por lo que se puede deducir que el aumento del PBI per cápita fue principalmente por el crecimiento de la economía del país, ya que el crecimiento poblacional tiene una tendencia decreciente. La variación del IPC en el periodo 2000 - 2014 fue de 42,75\%. Los precios internacionales han incidido en la inflación importada de manera muy alta en los años 2003, 2004 y 2008, pero de manera muy baja en el año 2009. En el periodo 2003 - 2012, el rubro formación bruta de capital que está dentro de los gastos de capital del gobierno general se ha duplicado en importancia respecto al PBI. En el año 2011 tuvo una caída de 18\%, y a partir de 2014 ha tenido una tendencia decreciente o negativa. El desempeño de la ejecución de lo presupuestado entre los años 2000 y 2009 ha sido muy errático. Por ejemplo, en el año 2004 el PIE o el devengado fue mayor que el PIM, es decir se gastó más de lo que se presupuestó, lo cual refleja la eficacia presupuestal o el desempeño de los funcionarios públicos o el desempeño institucional.

Hasta el año 2002 la cantidad de artículos científicos publicados fueron muy bajos y su crecimiento casi fue nulo. Solo a partir del año 2003 empieza a crecer la cantidad de artículos publicados llegando a ser en el 2014 casi seis veces más que el año 2002. Pero aun así esta cantidad de artículos publicados es mucho menor como proporción del total de docentes universitarios que existen en el país.

La Tasa Bruta de matrícula del periodo en estudio ha sido de constante crecimiento, ya que en estos quince años ha aumentado en $11 \%$. Pero, muchas personas que estudiaron solamente hasta secundaria o parte de ella se han olvidado mucho de lo aprendido, es decir, existe una discontinuidad lo que linda con el analfabetismo. En este caso según el Instituto Peruano de Economía (2015), Lima tenía 2.3\% de analfabetos, mientras que Huánuco poseía 16\% de analfabetos. En el año 2014 se puede establecer que una persona de cada cuatro que integran la PEA tiene educación superior o está capacitada en instituciones universitarias, tecnológicas o pedagógicas. El promedio de vida de un peruano ha aumentado en este periodo de estudio. Son cinco años más que vive un peruano en promedio, lo que significa que su salud ha mejorado en comparación al 2000, cuando ya terminaba el gobierno de Fujimori. Pero existe una diferencia entre las mujeres y los hombres, pues mientras que un hombre o varón tenía en el año 2000 una esperanza de vida de 68 años, una mujer tenía una esperanza de vida de 73 años. Finalmente, se analiza el costo de registrar una empresa, que se normaliza presentándolo como un porcentaje del Ingreso Nacional Bruto (INB) per cápita. Del periodo de estudio, el costo para establecer una empresa en el Perú disminuyó 33.8\%. Este desempeño tiene que ver mucho con los acuerdos comerciales que ha suscrito el Perú y con la flexibilización de la burocracia. 
Se aplicó los siguientes rezagos: Con el primer rezago de las variables (X1A), (X1B), (X2A), (X5A), (X5B) y X7. Y con el segundo rezago de la variable inflación o IPC (X2B). La probabilidad del t estadístico de todas las variables independientes son menores a 0.05 , por lo que, inicialmente podemos decir, que el modelo está correctamente especificado o construido.

Tabla 2. Contrastación de la Hipótesis General

\begin{tabular}{ccccc}
\hline & Coefficient & Std. Error & t-Statistic & Prob. \\
\hline C(2) & -1.009157 & 0.050489 & -19.98785 & 0.0025 \\
C(3) & -0.047612 & 0.004807 & -9.903692 & 0.0100 \\
C(4) & 0.443962 & 0.032360 & 13.71949 & 0.0053 \\
C(6) & 2.783113 & 0.124312 & 22.38820 & 0.0020 \\
C(7) & -0.032743 & 0.005221 & -6.271915 & 0.0245 \\
C(8) & -0.113984 & 0.006188 & -18.41897 & 0.0029 \\
C(9) & -0.151413 & 0.017492 & -8.656154 & 0.0131 \\
C(10) & -1.496311 & 0.081187 & -18.43043 & 0.0029 \\
C(11) & 0.026493 & 0.002084 & 12.71535 & 0.0061 \\
C(12) & 1.393743 & 0.063400 & 21.98324 & 0.0021 \\
& -0.036067 & 0.005174 & -6.971097 & 0.0200 \\
R-squared & 0.999983 & Mean dependent var & 10.04190 \\
Adjusted R-squared & 0.999897 & S.D. dependent var & 0.124492 \\
S.E. of regression & 0.001265 & Akaike info criterion & -10.68663 \\
Sum squared resid & $3.20 \mathrm{E}-06$ & Schwarz criterion & -10.20859 \\
Log likelihood & 80.46307 & Hannan-Quinn criter. & -10.78488 \\
Durbin-Watson stat & 3.236307 & & & \\
\hline
\end{tabular}

Estimation Command

LS LOG $(\mathrm{Y})=\mathrm{C}(2) * \operatorname{LOG}(\mathrm{X} 1 \mathrm{~A}(-1))+\mathrm{C}(3) * \mathrm{LOG}(\mathrm{X} 1 \mathrm{~B}(-1))+\mathrm{C}(4) * \mathrm{LOG}(\mathrm{X} 2 \mathrm{~A}(-1))+$ $\mathrm{C}(5) * \mathrm{LOG}(\mathrm{X} 2 \mathrm{~B}(-2))+\mathrm{C}(6) * \mathrm{LOG}(\mathrm{X} 3 \mathrm{~A})+\mathrm{C}(7) * \mathrm{LOG}(\mathrm{X} 3 \mathrm{~B})+\mathrm{C}(8) * \mathrm{LOG}(\mathrm{X} 4)+$ $\mathrm{C}(9) * \mathrm{LOG}(\mathrm{X} 5 \mathrm{~A}(-1))+\mathrm{C}(10) * \mathrm{LOG}(\mathrm{X} 5 \mathrm{~B}(-1))+\mathrm{C}(11) * \mathrm{LOG}(\mathrm{X} 6)+\mathrm{C}(12) * \mathrm{LOG}(\mathrm{X} 7(-1))$ 


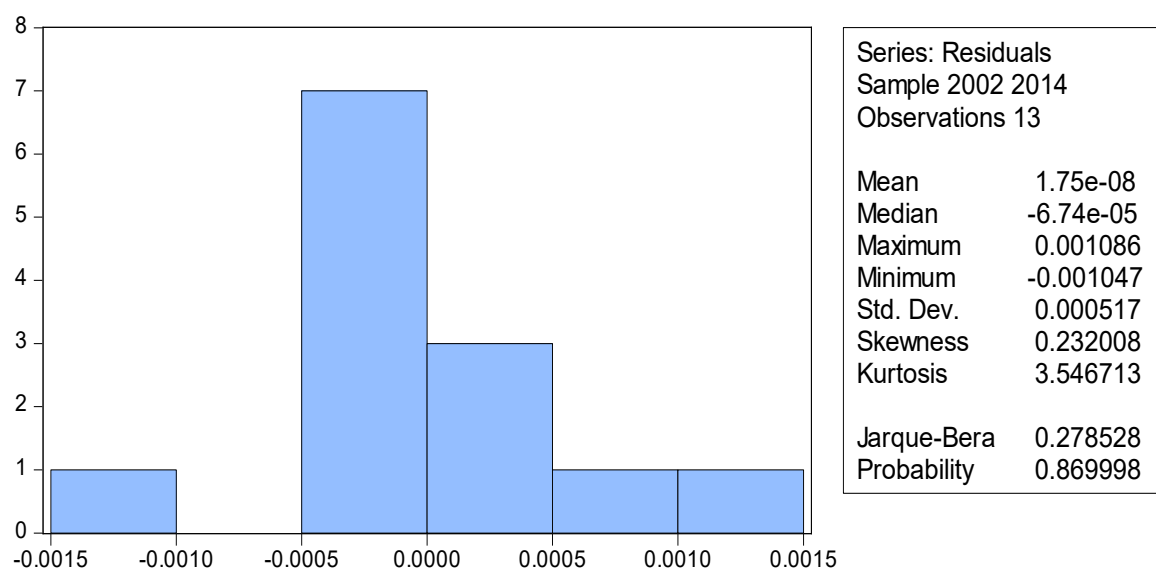

Figura 1. Normalidad del modelo

Date: 08/16/16 Time: 21:44

Sample: 20022014

Included observations: 13

\begin{tabular}{|c|c|c|c|c|c|c|}
\hline Autocorrelation & Partial Correlation & & $\mathrm{AC}$ & PAC & Q-Stat & Prob \\
\hline$\cdot\left|{ }^{* * *}.\right|$ & $\cdot||^{* * *} \cdot \mid$ & 1 & 0.366 & 0.366 & 2.1802 & 0.140 \\
\hline$\cdot * *|\cdot|$ &.$* * *|\cdot|$ & 2 & -0.206 & -0.393 & 2.9343 & 0.231 \\
\hline$\cdot|\cdot|$ & $\left.\cdot\right|^{* *} \cdot \mid$ & 3 & 0.002 & 0.343 & 2.9344 & 0.402 \\
\hline$\cdot|\cdot|$ & $\cdot * *|\cdot|$ & 4 & 0.036 & -0.322 & 2.9623 & 0.564 \\
\hline$\cdot *|\cdot|$ & $\left.\cdot\right|^{*} \cdot \mid$ & 5 & -0.122 & 0.145 & 3.3252 & 0.650 \\
\hline$\cdot *|\cdot|$ & $\cdot * *|\cdot|$ & 6 & -0.176 & -0.337 & 4.1834 & 0.652 \\
\hline$\cdot *|\cdot|$ & $\left.\cdot\right|^{*} \cdot \mid$ & 7 & -0.153 & 0.112 & 4.9447 & 0.667 \\
\hline$\cdot *|\cdot|$ & $* *|\cdot|$ & 8 & -0.118 & -0.295 & 5.4877 & 0.704 \\
\hline$\cdot *|\cdot|$ & $\left.\cdot\right|^{*} \cdot \mid$ & 9 & -0.114 & 0.098 & 6.1205 & 0.728 \\
\hline$\cdot|\cdot|$ & $\cdot *|\cdot|$ & 10 & -0.053 & -0.169 & 6.3039 & 0.789 \\
\hline$\cdot|\cdot|$ & $\left.\cdot\right|^{*} \cdot \mid$ & 11 & 0.021 & 0.085 & 6.3454 & 0.849 \\
\hline.$|\cdot|$ & $*|\cdot|$ & 12 & 0.017 & -0.138 & 6.4029 & 0.894 \\
\hline
\end{tabular}

Figura 2. Análisis de residuales

En cuanto a la probabilidad de F, tenemos que destacar que la estimación del modelo no tiene intercepto por ello no aparece en la tabla de regresión. El Durbin-Watson está fuera de la zona de no autocorrelación, por ello se muestra el correlograma o la autocorrelación parcial, cuyo gráfico es importante para esta explicación. Se han realizado muchas pruebas, lo cual nos indica que todos 
los indicadores son importantes. Respecto al coeficiente de determinación o de regresión $\mathrm{R}$ cuadrado ajustado, nos quiere decir que la gestión de la Competitividad Nacional del Perú está siendo explicada por las variables que representan los factores económicos-sociales en un 99.98\%, siendo estadísticamente significativa.

El Skewness está alrededor de cero y la Kurtosis alrededor de 3. El J-B muestra un valor probabilístico de 0.8699 que es mayor a 0.05 . Por lo que, no se rechaza la hipótesis nula de normalidad, comprobándose la asimetría y la curtosis de una distribución normal. Si elegimos a los coeficientes de las variables por importancia, podemos decir por un lado, que la variable inflación o índice de precios al consumidor tiene una mayor incidencia positiva que el resto de las variables, pues su valor es de 2.783; ello quiere decir, que si el IPC aumenta en $1 \%$ entonces la productividad nacional del Perú aumentará en $2.78 \%$. Mientras que por otro lado, tenemos que la variable población activa con educación superior tiene la menor incidencia, pues su valor es de 0.0265; ello quiere decir, que si la PEA activa con educación superior aumenta en $1 \%$ entonces la productividad nacional del Perú aumentará en $0.026 \%$. Asimismo, podemos destacar que la variable independiente que tiene mayor incidencia negativa es X5A o la inscripción escolar en el nivel secundario, pues su valor es -1.496; ello quiere decir, que si esta variable aumenta en $1 \%$ entonces la productividad nacional $(\mathrm{Y})$ disminuye en $1.5 \%$.

Tabla 3. Contrastación de Hipótesis Específicas

\begin{tabular}{|c|c|c|c|c|c|c|c|}
\hline $\begin{array}{l}\text { Hipótesis } \\
\text { Específica }\end{array}$ & variable & coeficiente & Prob. & $\mathrm{D}-\mathrm{W}$ & R- cuadrado & Normalidad & $\begin{array}{c}\text { Hipótesis } \\
\text { nula }\end{array}$ \\
\hline \multirow[t]{2}{*}{1} & $\mathrm{X} 1 \mathrm{~A}$ & 0.621668 & 0.0002 & 2.048765 & 0.975872 & $\mathrm{P}=0.717393$ & Rechazar \\
\hline & $\mathrm{X} 1 \mathrm{~B}$ & 0.068298 & 0 & & & & \\
\hline \multirow[t]{2}{*}{2} & $\mathrm{X} 2 \mathrm{~A}$ & 1.939763 & 0.0003 & 1.352931 & 0.720613 & $\mathrm{P}=0.872246$ & Rechazar \\
\hline & $\mathrm{X} 2 \mathrm{~B}$ & -1.706997 & 0.032 & & & & \\
\hline \multirow[t]{2}{*}{3} & $\mathrm{X} 3 \mathrm{~A}$ & 0.214208 & 0.0002 & 1.255883 & 0.849383 & $\mathrm{P}=0.634791$ & Aceptar \\
\hline & $\mathrm{X} 3 \mathrm{~B}$ & -0.100847 & 0.031 & & & & \\
\hline 4 & $\mathrm{X} 4$ & 0.233492 & 0 & 2.142372 & 0.972174 & $\mathrm{P}=0.585532$ & Aceptar \\
\hline \multirow[t]{2}{*}{5} & $\mathrm{X} 5 \mathrm{~A}$ & 2.33864 & 0 & 2.125921 & 0.876492 & $\mathrm{P}=0.649536$ & Aceptar \\
\hline & $\mathrm{X} 5 \mathrm{~B}$ & -0.146973 & 0.0008 & & & & \\
\hline 6 & X6 & 7.171917 & 0 & 1.829339 & 0.965702 & $\mathrm{P}=0.35168$ & Rechazar \\
\hline 7 & $\mathrm{X} 7$ & -0.427072 & 0.0004 & 1.503636 & 0.829892 & $\mathrm{P}=0.806759$ & Rechazar \\
\hline
\end{tabular}




\section{DISCUSIÓN}

Como menciona Porter (1991), la competitividad es la productividad (valor por unidad de insumo) en la que una nación, región o clúster usa sus recursos humanos y naturales. La productividad es el valor del rendimiento de una unidad de mano de obra o de capital. Es decir, el PBI entre la PEA ocupada. A partir de ello podemos relacionar lo expresado por el International Institute for Management Development (2015), respecto a la competitividad la cual la resume a "cómo una nación gestiona la totalidad de sus recursos y capacidades para aumentar la prosperidad de sus habitantes. La contrastación de la hipótesis general nos indica que por un lado, los factores que inciden positivamente sobre la variable dependiente son:

1) El desempeño económico a través de las variables $\mathrm{PBI}$ per cápita $(X 2 \mathrm{~A})$ e inflación (X2B).

2) La educación a través de la variable población activa con educación superior (X5B).

3) La salud a través de la variable esperanza de vida al nacer (X6).

Y que por otro lado, tenemos los factores que inciden negativamente, siendo estos:

1) La infraestructura a través de las variables (X1A) y (X1B).

2) La institucionalidad a través de las variables inversión per cápita del gobierno nacional y eficacia presupuestal en inversiones del gobierno nacional.

3) La innovación a través de la variable artículos en publicaciones científicas y técnicas.

4) La educación a través de la variable inscripción escolar en el nivel secundario (X5A).

5) El clima de negocios representado por la variable (X7).

Por consiguiente, si en el caso que todas las variables aumentaran en 1\% entonces el resultado final sería que la gestión de la competitividad (o la productividad) sería positivo o eficiente.

El modelo de regresión o especificación final muestra que eventos pasados inciden sobre los eventos futuros, como lo advierte Wooldridge (2010) el tiempo es una dimensión importante en las bases de datos de series de tiempo. La mayor parte de las series de tiempo económicas y otras series de tiempo están relacionadas, a menudo fuertemente, con sus historias recientes. Se aprecia que con los rezagos de las variables X1A, X1B, X2A, X5A, X5B, X2B, y X7 el modelo 
está correctamente especificado. El factor desempeño económico a través de la variable inflación (IPC), ha sido y es el mejor aliado de la política económica del gobierno, pues esta variable está relativamente controlada por mucho tiempo de acuerdo a la meta inflacionaria. Mientras que el factor educación a través de la variable nivel secundario incide de manera negativa. Este factor es el que tiene mayor incidencia negativa, como se puede desprender del coeficiente $C(9)$ que es igual a -1.496 ; lo cual nos indica que la política educativa tiene debilidades históricas y culturales.

Según el objetivo planteado, hemos determinado la manera como inciden los factores económicos-sociales en el impulso de la gestión de la competitividad del Perú. Existen variables que no tienen incidencia relevante, tales como: la inversión per cápita del gobierno nacional, cuyo coeficiente C(6) es igual a -0.03274; y la variable población activa con educación superior, cuyo coeficiente $\mathrm{C}(10)$ es igual a 0.02649 .

En contraste con los antecedentes de investigación, en el cual el Instituto Peruano de Economía (2013) encuentra la relación positiva que existe entre competitividad y crecimiento del producto, no se contradice con los resultados del presente estudio ya que el crecimiento económico o desempeño económico de la nación peruana tiene una relación directa con la competitividad. Entre el 2000 y el 2014, la productividad nacional aumentó en 48,1\%; por lo que, el aumento anual promedio fue de $3,2 \%$, que no es significativo pero que ello debe reflejarse en el aumento del sueldo mínimo vital.

\section{CONCLUSIONES}

La contrastación de la hipótesis general, nos da como resultado que los factores desempeño económico, educación a través de la variable población activa con educación superior; y salud, tienen incidencia positiva en la variable dependiente. Asimismo, los factores infraestructura, institucionalidad, innovación a través de la variable artículos en publicaciones científicas y técnicas, educación a través de la variable inscripción escolar en el nivel secundario, y clima de negocios; tienen incidencia negativa sobre la variable dependiente. Considerando los objetivos específicos tenemos las siguientes conclusiones:

La importancia de la incidencia del factor infraestructura no es muy significativa, porque solamente la variable hogares que se abastecen de agua tiene un resultado positivo relativamente significativo. La influencia del factor desempeño económico sí es relevante. Si el PBI por habitante y la inflación aumentan en 1\% el efecto neto sería positivo. 
La incidencia del factor institucional no es muy significativa. Dado los resultados si la inversión per cápita del gobierno nacional y la eficacia presupuestal en inversiones del gobierno nacional aumentan en la misma proporción, el efecto neto sería positivo.

El factor innovación no tiene una importante incidencia. Se debe a que la variable artículos en publicaciones científicas y Técnicas ha aumentado en forma consistente, pero no relevante.

La influencia del factor educación si es significativamente importante. La variable inscripción escolar en el nivel secundario tiene mayor importancia que la variable población activa con educación superior. El factor salud sí contribuye de manera muy importante, y el impacto del factor clima de negocios es de manera indirecta. Podemos señalar que la mayor fortaleza de la gestión de la competitividad nacional de este periodo, fue el desempeño económico y su mayor debilidad es la educación y en parte la infraestructura.

\section{AGRADECIMIENTOS}

A la Universidad Nacional Mayor de San Marcos por el apoyo académico, así como también a mi familia Cruz Ariza por su cariño y comprensión.

\section{REFERENCIAS BIBLIOGRÁFICAS}

Castro y Rivas-Llosa. 2005. Econometría Aplicada. Lima: CIUP.

Foro Económico Mundial. 2012. Índice Global de Competitividad. Suiza.

International Institute for Management Development. 2015. World Competitiveness Yearbook 2015. Suiza: Lausana.

Instituto Peruano de Economía. 2013. Índice de competitividad Regional (Incore) 2012. Lima.

Instituto Peruano de Economía. 2015. Índice de competitividad Regional (Incore) 2015. Lima.

Porter, M. 1991. La ventaja competitiva de las naciones. Buenos Aires: Vergara Editor S.A.

Wooldridge, J. M. 2010. Introducción a la econometría: Un enfoque moderno. 4a. edición. México, D.F.: Cengage Learning Editores, S.A. de C.V. Santa Fe C.P. 05349. 
|| José Miranda, Alfredo Marín, Darío Baño y Luis Hidalgo

Presentado: 06/04/2017

Aceptado: 10/05/2017

Correspondencia

Nelson Francisco Cruz Castillo

cruzc41@yahoo.es 